\title{
Editor de Textos Colaborativo Integrado ao Teleduc
}

\author{
José Alcir de Almeida Simões - alcirsimoes@yahoo.com.br \\ Vanessa Lindemann - vanessal@ulbra.br \\ Sistemas de Informação - ULBRA Cachoeira do Sul
}

\begin{abstract}
Resumo. O trabalho em grupo é importante porque possibilita que seus membros interajam, debatam diferentes pontos de vista e compartilhem o conhecimento.Este artigo apresenta o editor de textos colaborativo desenvolvido, integrado ao ambiente virtual de aprendizagem Teleduc. O objetivo é oferecer um espaço interativo, contribuindo para um efetivo trabalho em grupo, com a finalidade específica de produ ção coletiva de textos. Os resultados apresentados neste artigo referem-se à primeira versão do editor proposto no presente trabalho.

Palavras-chaves: editor de textos colaborativo, colaboração, Teleduc.
\end{abstract}

\section{Colaborativo Publisher of Texts Integrated to the Teleduc}

\begin{abstract}
The work in group is important because it makes possible that its members interact, debate different points of view and share the knowledge. This article presents the colaborativo publisher of texts developed, integrated to the virtual environment of learning Teleduc. The objective is to offer an interactive space, contributing for an effective work in group, with the specific purpose of collective production of texts. The results presented in this article mention the first version to it of colaborativo publisher of texts considered in the present work.
\end{abstract}

Keywords: colaborativo publisher of texts, collaboration, Teleduc.

\section{Introdução}

É cada vez mais comum a existência de grupos de trabalho, tanto na educação quanto nas organizações em geral. Com os avanços da tecnologia, essa forma detrabalho começou a ser suportada pelo computador, o que motivou a criação de áreas de pesquisa como a CSCW (Computer Supported Cooperative Work) e CSCL (Computer Supported Collaborative Learning). Essas áreas descrevem como devem ser desenvolvidas as ferramentas groupware, utilizadas para auxiliar o trabalho em grupo através do uso do computador.

Na educação, o trabalho em grupo é importante porque possibilita que os alunos interajam, debatam diferentes pontos de vista e compartilhem o conhecimento. Os membros do grupo têm o retorno de seus colegas, o que facilita a identificação precoce de falhas em seu raciocínio e, juntos, podem buscar informações que auxiliem na resolução dos problemas. Essa forma de trabalho exige uma organização colaborativa, onde a comunicação, a coordenação e a cooperação predominam.

Entretanto, acostumados com o paradigma de controle condicionado pela escola, onde a comunicação é vertical e as tarefas são realizadas individualmente, os alunos podem encontrar obstáculos para trabalhar em grupo. A cooperação entre os membros do grupo, por exemplo, pode ser prejudicada quando um de seus componentes acredita ser mais capaz que os demais para a execução das tarefas e/ou por falta de interesse de um ou mais integrantes do grupo. A comunicação pode falhar quando um ou mais membros do grupo é tímido e não se sente à vontade para colaborar ou, ao contrário, quando um componente se torna líder e acaba coagindo a participação dos demais. Essa liderança, por outro lado, pode ser importante para o fator coordenação, garantindo a organização dos membros do grupo para a execução das tarefas e, conseqüentemente, um bom desempenho no trabalho. 
Prover auxílio para o desenvolvimento de trabalhos em grupo, resolvendo alguns dos problemas apontados anteriormente, é um dos objetivos da utilização de editores colaborativos na educação. Através desses editores, os membros de um grupo têm acesso à versão atualizada e ao histórico do objeto que está sendo criado e podem participar da execução do trabalho no mesmo nível dos colegas. Além disso, o professor pode verificar as ações realizadas por cada membro do grupo, o que facilita o processo de avaliação.

Porém, a utilização de um editor colaborativo não atende a todos os requisitos necessários para o bom desenvolvimento e a avaliação de um trabalho em grupo. Os editores costumam abordar apenas o aspecto da edição de um objeto, não se preocupando em facilitar o gerenciamento do trabalho através de aspectos como a comunicação e a coordenação dos membros do grupo. A interação dos componentes do grupo costuma acontecer através de ferramentas groupware, como sala de bate-papo e correio eletrônico, que não são integradas aos editores, dificultando o gerenciamento das atividades do trabalho por parte dos alunos e impedindo que o professor tenha acesso a estas informações.

Diante desse contexto, propõe-se o desenvolvimento de um editor de textos colaborativo via web, integrado ao ambiente virtual de aprendizagem Teleduc, para propiciar a colaboração entre os membros de um grupo, provendo tanto aspectos de cooperação, quanto de comunicação e coordenação.

Este artigo está organizado da seguinte forma: a seção 2 é dedicada à fundamentação teórica e apresenta o ambiente virtual de aprendizagem Teleduc, ao qual o editor de textos colaborativo, desenvolvido no presente trabalho, foi integrado; a seção 3 descreve a metodologia e os resultados são apresentados na seção 4; considerações finais e perspectivas futuras são abordadas na seção 5 .

\section{Fundamentação Teórica}

A fundamentação teórica deste artigo aborda questões referentes ao trabalho em grupo, descreve o Modelo 3C e apresenta o Teleduc - Ambiente Virtual de Aprendizagem.

2.1 CSCW, CSCL e Groupware Nas últimas décadas, percebe-se o aumento no tamanho e na complexidade das tarefas executadas nas organizações. Além disso, o tempo disponibilizado para a execução dessas tarefas tem diminuído, dificultando que elas sejam realizadas por apenas uma pessoa. Isso motivou a formação de grupos de trabalho, onde pessoas com diferentes conhecimentos cooperam umas com as outras para aumentar a produtividade das organizações. Também é notável que o computador tem sido cada vez mais utilizado nas organizações, tanto para tarefas simples como, por exemplo, formatar um relatório, quanto no uso de softwares específicos para apoiar uma decisão ou obter informações dos clientes.

Para atender a essas mudanças, surgiu, em meados dos anos 70, a área de pesquisa chamada Automação de Escritório, que tinha como foco o aumento da produtividade das organizações. Os primeiros esforços dessa área foram permitir o acesso simultâneo de membros de um grupo de trabalho a aplicações que antes eram apenas mono-usuário (CARNEIRO, GELLER e TAROUCO, 2002). A partir dessas pesquisas, evidenciou-se a necessidade de estudar o comportamento desses grupos ao desempenhar uma atividade conjunta, fazendo com que a Automação de Escritório fosse sendo substituída gradativamente pela área de CSCW (Computer Supported Cooperative Work).

A sigla CSCW foi publicamente lançada em 1984, como título do seminário organizado por Paul Cashman e Irene Greif que reuniu pesquisadores das várias disciplinas compreendidas pelo tema (SANTOS, 1998). A CSCW foi definida como a disciplina científica que descreve como desenvolver aplicações groupware, isto é, como definir e ampliar o software e o hardware para que esses possam suportar e ampliar o trabalho em 
grupo. Seu objetivo é fazer com que o resultado obtido pelo grupo seja maior que a soma das contribuições individuais dos componentes (ROSA, 2005).

A partir dos estudos da área de CSCW, surgiu uma adaptação para a área da educação que ficou conhecida pela sigla CSCL (Computer Supported Collaborative Learning). Esta área, considerada por alguns autores como sendo uma subdivisão da CSCW, visa a proporcionar o aprendizado de um grupo de pessoas através do uso do computador. A Tabela 1 apresenta as principais diferenças entre as áreas de CSCW e CSCL.

\begin{tabular}{l|l}
\multicolumn{1}{c}{ Tabela $1-$ Principais diferenças entre CSCW e CSCL } \\
\hline CSCW & CSCL \\
\hline $\begin{array}{l}\text { Tende a focalizar sua atenção nas técnicas de } \\
\text { comunicação. }\end{array}$ & $\begin{array}{l}\text { Tende a focalizar sua atenção no que está a ser } \\
\text { comunicado. }\end{array}$ \\
\hline $\begin{array}{l}\text { Está sendo utilizada principalmente na área } \\
\text { empresarial. }\end{array}$ & $\begin{array}{l}\text { Está sendo explorada em ambientes } \\
\text { educacionais. }\end{array}$ \\
\hline $\begin{array}{l}\text { Tem como finalidade facilitar a comunicação } \\
\text { e a produtividade do grupo. }\end{array}$ & $\begin{array}{l}\text { Tem como finalidade sustentar a aprendizagem } \\
\text { eficaz do grupo. }\end{array}$ \\
\hline
\end{tabular}

Fonte: Tecnologia Educativa, 2000.

As tecnologias geradas pela área de CSCL são importantes para desenvolver o espírito de equipe nos alunos, que é indispensável para a realização de um trabalho em grupo. Os membros de um grupo devem unir-se em torno de uma tarefa comum, buscando compartilhar seus conhecimentos e esfornçando-se, colaborativa e/ou cooperativamente, no sentido de atingir, no coletivo, as metas estabelecidas. Na educação presencial o trabalho em grupo favorece a interação social do aprendiz. Na educação à distância, além da interação social, que ocorre de forma virtual, o trabalho em grupo pode tornar-se fator a mais de motivação e compromisso frente aos desafios estabelecidos pela aprendizagem, principalmente no sentido de enfatizar a dimensão coletiva na vida dos alunos (MARTINS e AXT, 2004). Em ambas as modalidades de ensino, o trabalho em grupo motiva os alunos a serem sujeitos ativos no processo de ensino-aprendizagem, atendendo o novo paradigma da educação.

O termo groupware é muitas vezes utilizado como sinônimo de CSCW ou CSCL, mas há uma visível diferença. O termo groupware, criado em 1978, nasceu da união das palavras group e software e foi definido como sistemas baseados em computador que apóiam grupos de pessoas envolvidas em uma tarefa (ou objetivo) comum e que proporcionam uma interface para o ambiente compartilhado (CARNEIRO, GELLER e TAROUCO, 2002). Portanto, enquanto o CSCW e o CSCL são as áreas de pesquisa, os groupware são as tecnologias geradas por estas áreas e que dão suporte ao trabalho ou aprendizado cooperativo/colaborativo.

Desde a criação do termo groupware, ele vem sofrendo modificações quanto a sua utilização. Quando foi criado, o termo era usado para aplicações que, de alguma forma, podiam apoiar o trabalho em grupo. Atualmente, o termo também é utilizado para designar os ambientes que integram mais de uma aplicação groupware (NITZKE e FRANCO, 2002).

No presente trabalho, as aplicações individuais são chamadas de ferramentas groupware, enquanto que os sistemas que apresentam um conjunto dessas ferramentas integradas são designados como ambientes virtuais de aprendizagem.

\subsection{O Modelo 3C}


As ferramentas groupware são sistemas que buscam apoiar o trabalho de um grupo na realização de uma tarefa e, quando integradas umas com as outras, formam um ambiente colaborativo. Para que um ambiente seja realmente propício à colaboração, é necessário que suporte os aspectos de comunicação, cooperação e coordenação. A combinação desses requisitos ficou conhecida como Modelo 3C (FUCKS, RAPOSO e GEROSA, 2003; PIMENTEL et al., 2005). Nesse modelo, os aspectos estão relacionados e as ferramentas groupware atendem a um ou mais deles, como mostra a Figura 1.

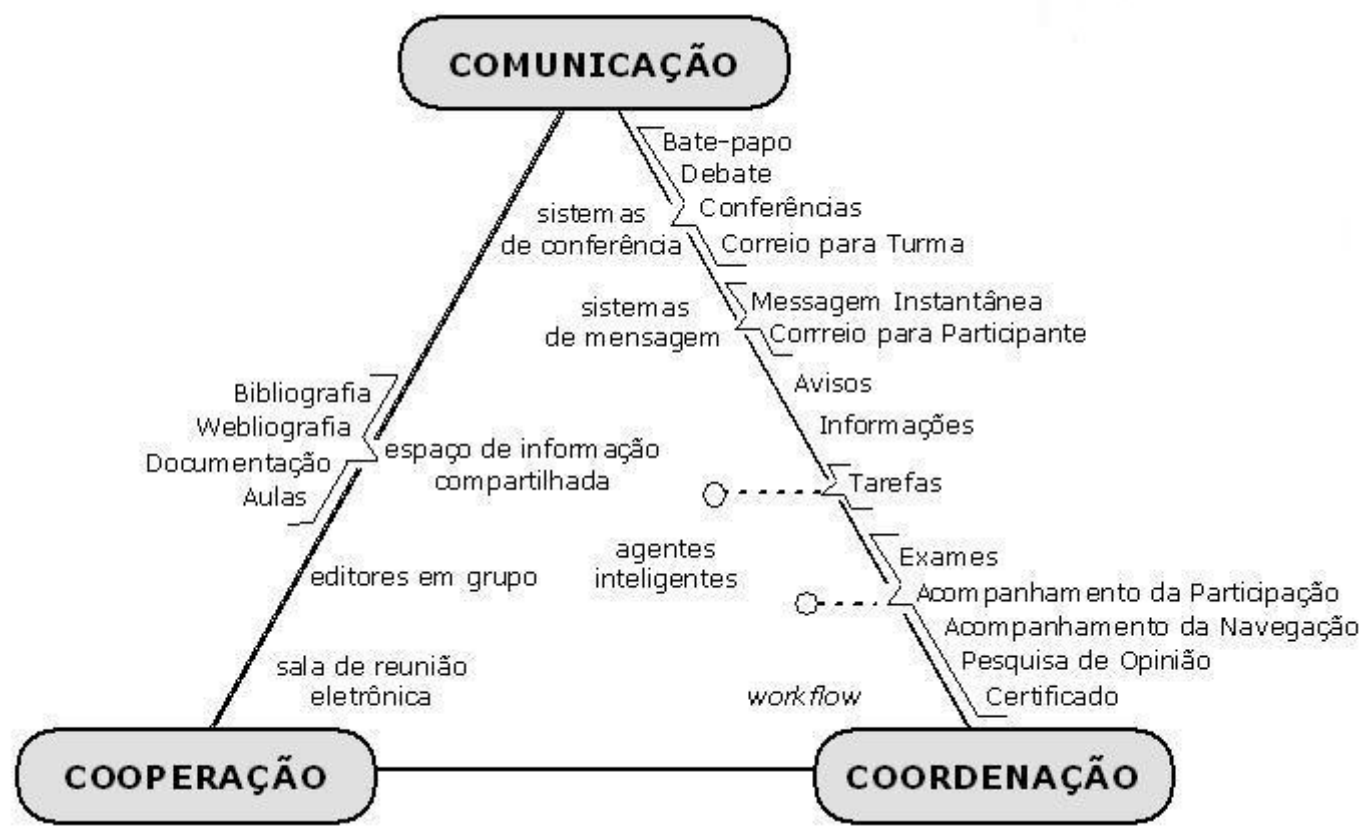

Figura 1 - Modelo 3C e groupwares (PIMENTEL et al., 2005)

Comunicação: o apoio à comunicação é essencial nos sistemas colaborativos, pois proporciona a interação entre os membros de um grupo. É importante que um ambiente virtual de aprendizagem possibilite que os integrantes do grupo troquem idéias, informações e documentos para que suas tarefas sejam realizadas mais eficientemente (ROSA, 2005). Essa comunicação pode ser disponibilizada de modo síncrono (ao mesmo tempo), quando se deseja ter maior velocidade na interação. Outro tipo de comunicação é a assíncrona (em tempos diferentes), que é mais utilizada quando se deseja promover a reflexão do integrante.

Cooperação: a cooperação está diretamente ligada à comunicação, pois para atingi-la é necessário que se dê suporte ao compartilhamento de informações de forma que ofereça um contexto sempre atual ao grupo e notifique as ações realizadas pelos seus integrantes. Com isso, o ambiente se torna propício à cooperação, já que possibilita que cada membro do grupo esteja ciente do que deverá executar.

Coordenação: para haver um melhor aproveitamento do sistema colaborativo, é importante que ele possibilite que os componentes de um grupo estejam coordenados e isso geralmente é feito através do compartilhamento de informações. Assim, é possível minimizar problemas como a execução de tarefas repetidas realizadas por diferentes integrantes do grupo. Além disso, a coordenação é um ponto essencial para que o grupo se mantenha informado sobre prazos que devam ser cumpridos.

\subsection{Teleduc: Ambiente Virtual de Aprendizagem}

O Teleduc é um ambiente virtual de aprendizagem utilizado como ferramenta de apoio em aulas presenciais e no ensino a distância (EAD). $\mathrm{O}$ ambiente possui várias 
ferramentas groupware, dentre elas, fórum de discussão, sala de bate-papo, mural, portfólio e correio eletrônico.

O Teleduc foi desenvolvido por pesquisadores do Núcleo de Informática Aplicada à Educação (NIED) e pelo Instituto de Computação (IC) da Universidade Estadual de Campinas (UNICAMP) (TELEDUC, 2001). Sua primeira versão como software livre foi lançada em fevereiro de 2001. Em março de 2002, foi lançada a versão 3.0, com a reestruturação e a otimização do ambiente e o suporte a múltiplas línguas para atender a

demanda de uso internacional do ambiente.

O sistema foi codificado utilizando, principalmente, a linguagem de programação PHP (PHP Hypertext Preprocessor). Outras linguagens utilizadas foram a Perl para a ferramenta de bate-papo e o Java para a ferramenta intermap. Para o gerenciamento do banco de dados foi utilizado o MySQL. Para a sua instalação é necessária a configuração de um servidor web na plataforma Linux.

O Teleduc tem sido bastante utilizado por universidades, instituições públicas e privadas. No cadastro de instituições usuárias do ambiente há universidades como a Universidade Federal do Rio Grande do Sul (UFRGS), a Universidade de Brasília (UnB), a Pontifícia Universidade Católica de São Paulo (PUC-SP) e a Universidade Luterana do Brasil (ULBRA). Ainda possui cadastradas instituições públicas como a Secretária da Fazenda do Estado de São Paulo e privadas como a Conectiva.

\section{Metodologia}

Para a consecução das atividades propostas pela pesquisa, aqui apresentada, inicialmente, foi realizada a fundamentação teórica do trabalho, que abordou aspectos necessários para o seu desenvolvimento. Um dos temas abordados foram as diferenças entre os termos CSCW, CSCL e groupware. Nessa fase, também foram escolhidos os editores colaborativos que foram avaliados para identificar funcionalidades importantes nessas ferramentas e que seriam consideradas no editor desenvolvido no trabalho. Outro tema importante estudado foi o conceito de Modelo 3C, que ajudou na identificação de alguns aspectos que podem ser melhorados no trabalho em grupo quando este é realizado com o suporte de um editor colaborativo integrado a um ambiente virtual de aprendizagem.

$\mathrm{Na}$ segunda etapa do trabalho, foi realizada uma avaliação dos editores colaborativos EquiText (EQUITEXT, 2001) e Twiki (TWIKI, 1998). O editor Co-link (COLINK, 2003) também seria avaliado, mas a ferramenta não estava disponível para teste na época da execução dessa etapa e não se obteve resposta aos contatos feitos com aqueles que desenvolveram a ferramenta. Para a análise desses editores, foi elaborado um instrumento padrão de avaliação, com dezoito questões sobre funcionalidades consideradas importantes para que essas ferramentas promovam a colaboração. Na tabulação dos resultados, foi utilizada a escala de Likert, que possibilita que cada questão possua um dos cinco valores possíveis: -2 (descordo totalmente), -1 (descordo parcialmente), 0 (não foi possível avaliar), 1 (concordo parcialmente) e 2 (concordo totalmente). O objetivo dessa avaliação foi analisar a possibilidade de integrar um dos editores citados ao ambiente virtual de aprendizagem Teleduc.

Da análise dos resultados da avaliação realizada, destaca-se como vantagem do EquiText o fato do editor possibilitar que diferentes trechos do texto sejam editados ao mesmo tempo por diferentes integrantes do grupo. Entretanto, a mesma característica, que possibilita essa funcionalidade, dificulta a inserção e a edição do texto, já que essas ações precisam ser realizadas separadamente em cada trecho (parágrafo) do texto. Do TWiki, aponta-se o histórico do texto como ponto positivo. São mantidas várias versões do texto com informações sobre as atualizações realizadas. Porém, o histórico é de difícil análise, principalmente, quando o texto é extenso e/ou há várias versões do mesmo. Considerando que (a) os editores colaborativos avaliados não oferecem todas as características desejadas e 
que (b) modificar qualquer um dos editores estudados seria complicado, propôs-se o desenvolvimento de um editor colaborativo novo, integrado ao ambiente virtual de aprendizagem Teleduc.

O Teleduc foi escolhido por (a) possuir as ferramentas groupware necessárias para prover a colaboração; (b) ser um software livre, o que possibilita a manipulação do seu código fonte; (c) funcionar estritamente através da web; (d) estar codificado na mesma linguagem e utilizar o mesmo sistema gerenciador de banco de dados escolhidos para implementar o editor colaborativo proposto neste trabalho; e (e) já ser utilizado em várias instituições de ensino.

A instalação do ambiente virtual de aprendizagem escolhido configurou-se na terceira etapa do trabalho. Para instalar o Teleduc, foi necessário configurar um servidor web na plataforma Linux. Utilizou-se Apache versão 2.2.3 e a distribuição Linux escolhida foi a Kurumin, versão 6. Além disso, foram instaladas a linguagem de programação PHP e o sistema gerenciador de banco de dados MySQL, também necessários para o funcionamento do ambiente.

A fase de projeto do editor colaborativo, quarta etapa do trabalho, compreendeu a definição do layout da interface, a descrição das funcionalidades do sistema e a elaboração do Diagrama Entidade-Relacionamento (DER) utilizado para modelar o banco de dados do editor colaborativo implementado.

A quinta etapa do trabalho consistiu no desenvolvimento do editor colaborativo propriamente dito. Para implementar o editor, utilizou-se a linguagem de programação PHP e o sistema gerenciador de banco de dados MySQL. 4.1. Nessa etapa, foram realizados testes com o HTMLArea JS-RichEdit para analisar se o mesmo poderia ser utilizado na implementação do editor colaborativo desenvolvido. Esse componente permite que um texto seja formatado na web sem a necessidade do usuário conhecer a linguagem HTML (HyperText Markup Language). O componente foi desenvolvido com a linguagem JavaScript e sua utilização é permitida desde que não seja utilizado para fins comerciais e seja mantido o seu copyright (JS-RICHEDIT, 2007). O componente JS-RichEdit foi escolhido porque possui as principais funções para formatação e permite a inserção de figuras, links e tabelas no texto.

\section{Resultados}

O presente trabalho resultou em um editor de textos colaborativo, integrado ao ambiente virtual de aprendizagem Teleduc, para atender aos aspectos de comunicação, cooperação e coordenação do Modelo 3C que não são suportados com a utilização isolada de um editor colaborativo. A tela inicial do editor desenvolvido pode ser observada na Figura 2.

$\mathrm{Na}$ tela inicial do editor, são listados todos os textos cadastrados no curso em que o usuário ativo está logado, além de uma opção para que ele possa cadastrar um ou mais textos novos. Se o usuário for um aluno, os textos são divididos levando em consideração aqueles em que ele pode contribuir, ou seja, os textos nos quais ele é membro do grupo, e os textos de outros grupos, aos quais ele não tem acesso. Nos textos em que o aluno não tem acesso, há a opção para solicitar participação. No caso do usuário ser um professor, o acesso é permitido a todos os textos cadastrados. Além disso, esse tipo de usuário também tem acesso à opção de listagem de todas as versões de um determinado texto. 




Figura 2 - Tela inicial do editor desenvolvido, integrado ao Teleduc

Para cadastrar um texto, o usuário deve escolher um título e informar se este ficará acessível apenas aos componentes do grupo ou poderá ser visualizado ou editado por todos os integrantes do curso. No caso do acesso ficar restrito ao grupo, o usuário deve informar também quais serão os membros do grupo que terão acesso. É importante ressaltar que nessa escolha não são considerados os professores, já que eles têm acesso a todos os textos cadastrados no curso.

Na opção de visualização do texto, o usuário tem acesso a sua versão atual, como mostra a Figura 3. Acima do texto, à esquerda, há as opções "Editar", que possibilita a inserção ou edição do texto; e "Comentar", que permite a inserção de comentários sobre o texto, que ficam disponíveis aos demais membros do grupo. À direita, há as opções "Minha contribuição", que destaca todos os trechos que foram inseridos pelo usuário ativo; "Última Edição", onde são destacados os trechos do texto que foram incluídos na versão atual do documento; e "Texto Normal", que apresenta o texto sem nenhum tipo de destaque. Além dessas opções, quando o usuário é um professor há a opção "Todos", que indica a contribuição de cada um dos membros do grupo. Para isso, o sistema apresenta uma legenda onde são listados os membros do grupo que contribuíram com o texto e estes são associados a uma cor, utilizadas para destacar suas contribuições na versão ativa do documento (Figura $4)$. 


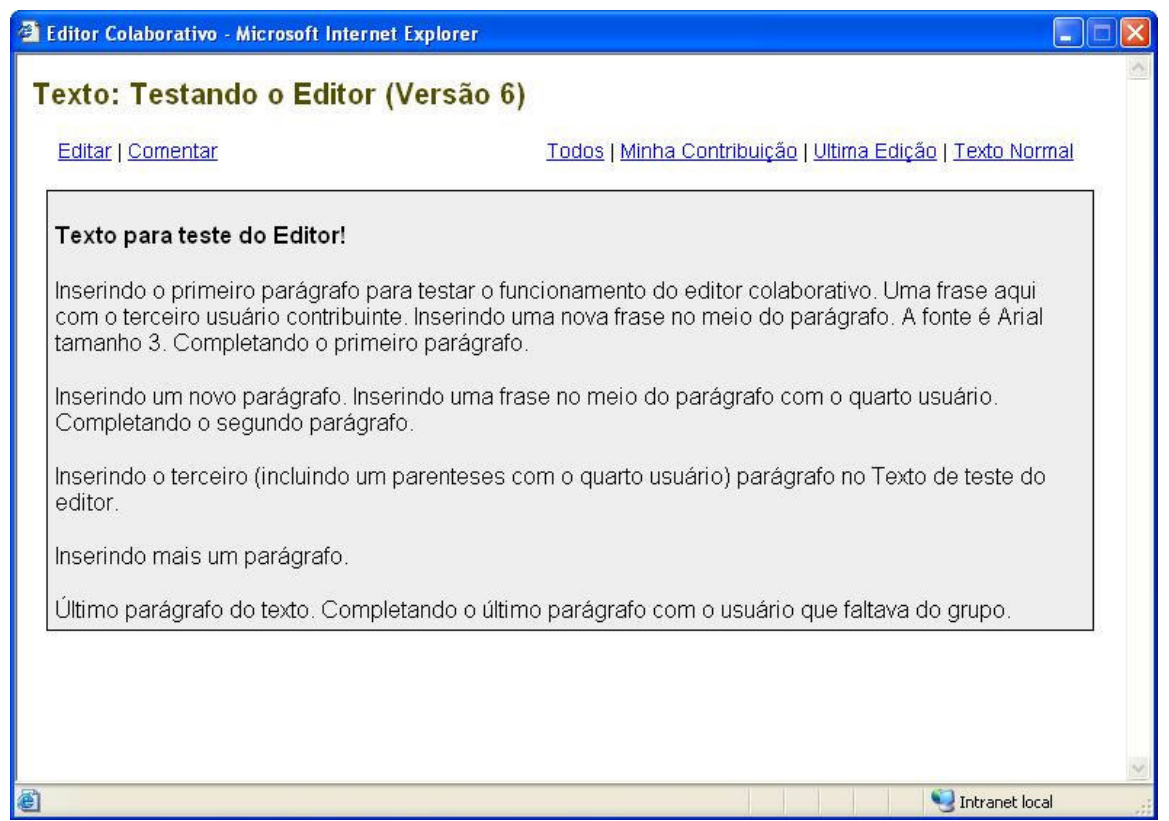

Figura 3 - Tela de visualização de um texto

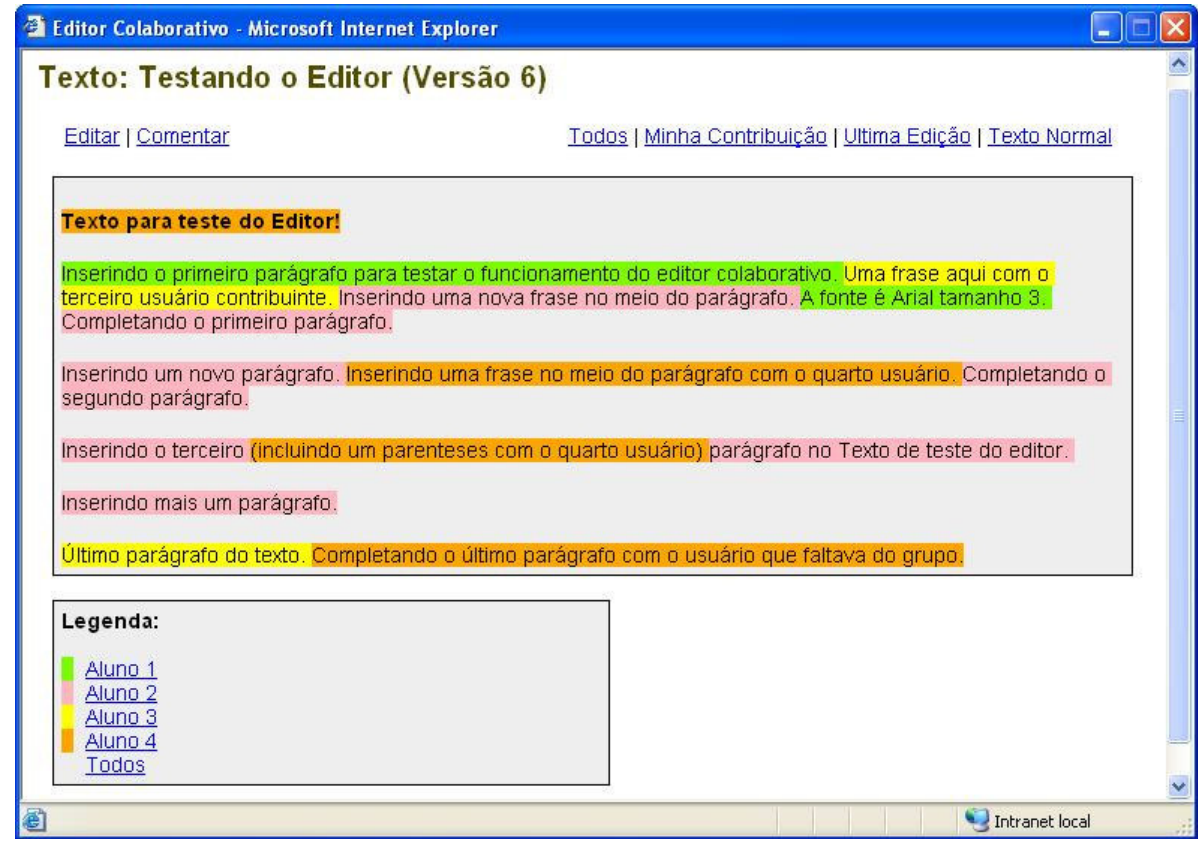

Figura 4 - Tela de visualização de um texto com contribuições de todos os usuários

Na legenda, o professor pode selecionar "Todos" para visualizar a contribuição de todos os membros do grupo, como ilustra a Figura 4, ou clicar sobre o nome de um dos alunos que compõem o grupo para visualizar apenas a sua contribuição no documento ativo. Essa funcionalidade do editor é importante pois a avaliação do professor, com relação à participação dos integrantes do grupo no texto criado, pode ser realizada de forma menos trabalhosa do que quando é necessário analisar todo o histórico do texto, como acontece nos editores avaliados no presente trabalho.

A opção "Editar" permite que um texto seja formatado através da utilização do componente JS-RichEdit, como mostra a Figura 5. Com isso, a formatação pode ser realizada sem que o usuário utilize tags HTML. 


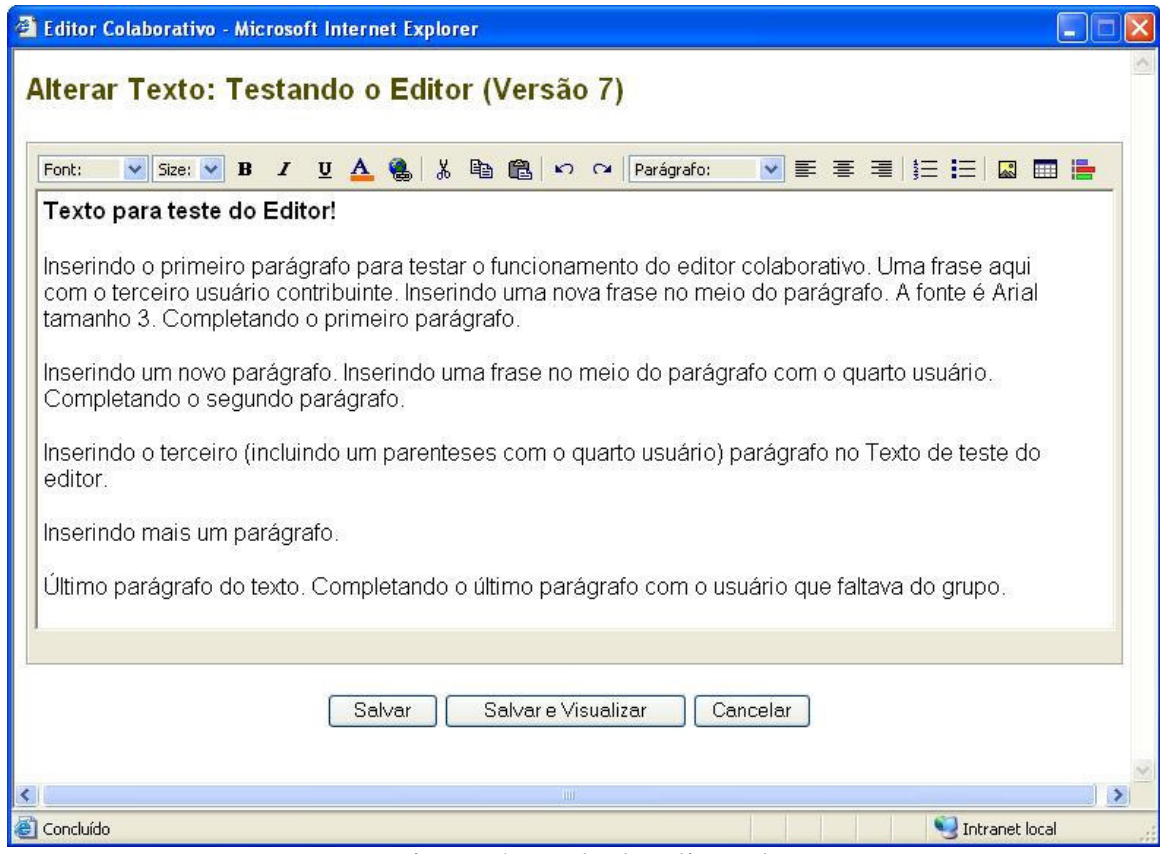

Figura 5 - Tela de edição de um texto

Outro diferencial do editor apresentado é o fato dele estar integrado ao ambiente virtual de aprendizagem Teleduc, que possibilita que os membros do grupo utilizem ferramentas groupware para melhorar a interação, de forma que o professor tenha acesso aos registros destas ações.

\section{Considerações Finais}

Uma das funções dos ambientes virtuais de aprendizagem é proporcionar facilidades de comunicação e expressão aos usuários, de forma articulada, para que o gerenciamento das ações possibilite o desenvolvimento de atividades em grupo, permitindo a confrontação e a negociação de diferentes pontos de vista, abrindo a um operar comum na direção das metas estabelecidas (MARTINS e AXT, 2004).

O presente trabalho pretende contribuir nesse sentido através do desenvolvimento de um ambiente propício à colaboração, considerando aspectos de cooperação, comunicação e coordenação. Com o editor de textos colaborativo desenvolvido, integrado ao ambiente virtual de aprendizagem Teleduc, pretende-se oferecer um espaço interativo, contribuindo para um efetivo trabalho em grupo, com a finalidade específica de produção coletiva de textos.

Os resultados apresentados neste artigo referem-se à primeira versão do editor de textos colaborativo desenvolvido. Essa versão está em processo de testes, realizados $10 \mathrm{com}$ turmas do curso de Sistemas de Informação, na ULBRA Cachoeira do Sul, para a realização de trabalhos em grupo propostos pelos professores em suas disciplinas. A intenção é verificar como pode se dar o uso do editor de textos colaborativo na educação. Os resultados desse estudo serão apresentados posteriormente, com maiores articulações teóricas. Por fim, resta salientar que outras funcionalidades já estão sendo implementadas e serão integradas à versão atual do editor, como, por exemplo, a possibilidade de criar ipertextos.

\section{Referências}


CARNEIRO, Mára Lúcia Fernandes; GELLER, Marlise; TAROUCO, Liane Margarida Rockenback. Groupware e os Ambientes para EAD. Informática na Educação: Teoria e Prática, v. 5, n. 2, p.11-21, 2002.

Co-Link. 2003. Disponível em: <http://www.co-link.org>. Acesso em: set. 2006. EquiText. 2001. Disponível em: <http://equitext.pgie.ufrgs.br>. Acesso em: set. 2006.

FUCKS, Hugo; RAPOSO, Alberto Barboas; GEROSA, Marcos Aurélio. Do Modelo de Colaboração 3C à Engenharia de Groupware. 2003. Disponível em: $<$ www.tecgraf.pucrio.br/publications/artigo_2003_colaboracao_3c_engenharia_groupware. pdf>. Acesso em: out. 2006.

JS-RICHEDIT. Disponível em: <http://gerd-tentler.de/tools/richedit>. Acesso em: fev. 2007.

MARTINS, Ademir da Rosa; AXT, Margarete. EccoLogos: autoria coletiva de documentos. Informática na Educação: Teoria e Prática, v. 7, n. 2, p. 39-49, 2004.

NITZKE, Júlio Alberto; FRANCO, Sérgio Roberto Kieling. Aprendizagem Cooperativa: utopia ou possibilidade? Informática na Educação: Teoria e Prática, v. 5, n. 2, p. 23-30, 2002.

PIMENTEL, Mariano; et al. AulaNet 3.0: desenvolvendo aplicações colaborativas baseadas em componentes 3C. 2005. Disponível em: <ritv.les.inf.pucrio. br/publicacoes/2005.WCSCW.Pimentel.pdf>. Acesso em: nov. 2006.

ROSA, Márcio. Groupware: um caminho para a cooperação. 2005. Disponível em: <http://www.frb.br/ciente/2005.1/BSI/ciente_v.1_bsi.rosa.pdf>. Acesso em: ago. 2006.

SANTOS, Adriana Delfino dos. Autoria Colaborativa: tendências. 1998. Disponível em:<http://www.dca.fee.unicamp.br/courses/IA368F/1s1998/Monografias/adriana/inde x.html. Acesso em: ago. 2006.

Tecnologia Educativa. Aprendizagem Colaborativa Assistida por Computador. 2000. Disponível em: <http://www.minerva.uevora.pt/cscl/>. Acesso em: set. 2006.

TelEduc. 2002. Disponível em: <http://teleduc.nied.unicamp.br/pagina/>. Acesso em: mai. 2006.

T-Wiki. 1998. Disponível em: <http://twiki.org>. Acesso em: set. 2006. 